\title{
El impacto en la vida laboral de estudiantes de Licenciatura en Educación y su experiencia al estudiar en modalidad virtual
}

\author{
doi: 10.33264/rpa.202001-10 \\ María Angélica Sanhueza Álvarez \\ Dirección de Educación Virtual, Universidad La República
}

\section{Resumen}

La presente investigación tiene por objetivo exponer cuáles son las repercusiones en la vida laboral de cincuenta estudiantes profesionales de diferentes regiones de Chile que estudiaron Licenciatura en Educación a través de la modalidad virtual en la Universidad La República. A la vez, considera las consecuencias en cuanto al manejo de los recursos virtuales para cumplir con los objetivos de la formación.

La metodología utilizada corresponde al análisis de las diferentes respuestas que se obtuvieron por parte de los actores involucrados aplicando una entrevista semiestructurada y se propone como una forma de validar la modalidad virtual, revisando sus barreras y facilitadores en la formación de un Licenciado en Educación.

Palabras clave: Licenciatura en Educación, Educación virtual, vida laboral, recursos virtuales.

\section{Abstract}

The present investigation aims to give knowledge about the repercussions on the working life of fifty student professionals from different regions of Chile who followed the Degree in Education course through the virtual platform of Universidad La República. At the same time, this investigation considers the consequences regarding the handling of the virtual resources to comply with the objectives of the course.

The methodology used corresponds to the analysis of the different answers that were obtained from the actors involved applying a semi-structured interview and it is proposed as a way to validate the virtual modality, reviewing its barriers and facilitators in the formation of a Degree in Education.

Keywords: Degree in Education, Virtual Education, working life, virtual resources. 


\section{Introducción}

En el contexto actual de la educación chilena, la Licenciatura en Educación se presenta como una alternativa que, si bien no entrega el título de profesor, permite habilitar a los profesionales que la siguen a trabajar en un establecimiento educacional. La autorización para ejercer docencia se otorgará preferentemente a quienes poseen título docente en una especialidad o nivel diferente o a quienes posean certificados de validación o competencias indiscutibles para ejercer docencia (art. 10 Decreto $\mathrm{N}^{\circ} 352$ ). Por otra parte, es posible encontrar a algunos estudiantes, participantes de esta investigación, que cursan la licenciatura, y deciden tomar esta formación con el fin de adquirir nuevos conocimientos, distintos a su formación de origen, y que no precisamente se desenvuelven laboralmente en establecimientos educacionales. Las realidades de estos estudiantes son diversas, pues pertenecen a diferentes lugares de Chile, con distintas características socio culturales y con un amplio rango etario. Considerando lo anterior, esta investigación pretende responder a estos cuestionamientos, y con ello aportar en la validación de esta formación y su modalidad.

Según las estadísticas del Ministerio de Educación de Chile (2017), confirman un incremento de la matrícula total en programas virtuales, "en el año 2016, el número de estudiantes en esta modalidad creció $21 \%$ respecto a 2015, totalizando 24.659, cifra que contrasta con los 8.291 que estaban matriculados en 2012". Cifras más actuales corroboran esta información según el Índice País Digital en el año 2017, “más de 12 millones de personas han usado Internet en el último tiempo, en una cifra equivale nte al $\mathbf{7 1}$ \% de la población nacional”. Según Cecilia Rojas, del centro IPLACEX en los últimos 5 años, la modalidad de educación a distancia creció un 141\%. Tan solo en el 2018, el alza fue de 25\%. Estos datos permiten visualizar la importancia de realizar esta investigación, y con ella validar la formación de los licenciados en educación y la modalidad no presencial.

Por otra parte, Néstor Milano, director ejecutivo de laborum.com, respecto al perfil del estudiante online señala (2018) que "Además de manejarse con las herramientas tecnológicas propias del e-learning, como aplicaciones y trabajo en línea, el estudiante debe ser capaz de organizar sus tiempos, desarrollar habilidades de autonomía y proactividad, y por supuesto, mostrarse receptivo al trabajo colaborativo". Lo anterior, será confrontado con los resultados de esta investigación. Este estudio se inicia en el mes de junio del año 2019. Si bien durante la formación existen instancias de comunicación con los participantes a través de la mensajería entre profesor y estudiantes, en esta oportunidad la comunicación ocurre por medio de una pantalla y es posible conversar en dos momentos: primero, a través de la "sesión de prueba", previo al Examen Final y luego, en el Examen Final formal de la 
asignatura. La primera instancia, es una conversación entre la coordinación académica y cada estudiante, donde se aclaran dudas en relación con el protocolo, la formalidad y al uso de la aplicación Meet, que corresponde a una aplicación de mensajería multiplataforma. Todo esto con el fin de que el estudiante sienta mayor seguridad respecto a lo que debe hacer y cómo debe hacerlo. En el momento formal del Examen Final de la asignatura Seminario de Grado, la Comisión Evaluadora, compuesta por el docente de dicha asignatura, un/a Representante de la Escuela de Educación, y la Presidenta de la Comisión, evalúan la presentación del estudiante.

Luego de esta evaluación, el proceso finaliza con unas preguntas dirigidas al estudiante, relacionadas con el proceso vivido, se produce entonces una conversación que se basa en dos tópicos: la utilidad de esta formación y el cómo se enfrenta cada uno de ellos a esta nueva modalidad de estudio, identificando facilitadores y barreras, lo que dará respuesta a esta investigación y sus interrogantes. De esta forma, se pretende mejorar aquellos aspectos menos desarrollados y fortalecer aquellos que han sido útiles para los estudiantes y sus procesos. A partir de las ideas planteadas, se desprenden las siguientes preguntas ¿cómo repercute esta formación y su modalidad no presencial en la vida laboral de los estudiantes que la siguen?, ¿cuáles fueron las mayores dificultades encontradas?

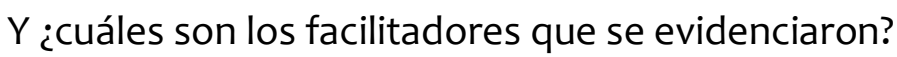

La investigación tiene como objetivo general: Conocer el impacto en la vida laboral de estudiantes que cursaron Licenciatura en Educación, en la Universidad La República, identificando barreras y facilitadores que evidenciaron en la modalidad virtual en que se lleva a cabo dicha formación.

\section{Metodología}

De acuerdo con el tema central y los objetivos propuestos, se realiza una investigación de corte cualitativo, intentando comprender la realidad y profundizar en el conocimiento de ésta. El paradigma cualitativo es el que utiliza la recolección de datos sin medición numérica para descubrir o afinar preguntas de investigación en el proceso de interpretación.

El enfoque cualitativo se selecciona cuando el propósito es examinar la forma en que los individuos perciben y experimentan los fenómenos que los rodean, profundizando en sus puntos de vista, interpretaciones y significados (Sampieri, 2017, p. 358).

Angrosino M. (2012, p. 10) explica que la investigación cualitativa pretende acercarse al mundo de "ahí afuera" (no en entornos de investigación especializada como los 
laboratorios) y entender, describir y algunas veces explicar fenómenos "desde el interior" de varias maneras diferentes:

- Analizando las experiencias de los individuos o de los grupos. Las experiencias se pueden relacionar con historias de vida biográficas o con prácticas (cotidianas o profesionales); pueden tratarse analizando el conocimiento cotidiano, informes e historias.

- Analizando las interacciones o comunicaciones mientras se producen. Esto se puede basar en la observación o el registro de las prácticas de interacción y comunicación, y en el análisis de ese material.

- Analizando documentos (textos, imágenes, películas o música) huellas similares de las experiencias o interacciones.

A raíz de lo expuesto en los párrafos anteriores, surgieron los siguientes supuestos:

Se presume que la Licenciatura en Educación modalidad virtual, contribuye al desarrollo integral de los profesionales que la cursan, recibiendo herramientas adecuadas que les permiten desenvolverse en su labor en establecimientos educacionales, potenciando el desarrollo de las habilidades en relación con los estudiantes con quienes trabajan y con otros docentes respecto a conocimientos del quehacer pedagógico.

Se estima que la modalidad virtual, brinda las oportunidades para un adecuado manejo de los contenidos tratados en cada asignatura y en los respectivos módulos (clases) de éstas, otorgándoles herramientas a través de una metodología de estudio que responde a sus intereses y necesidades de manera íntegra.

\section{Participantes en la investigación}

Se analizan las respuestas de cincuenta estudiantes de Licenciatura en Educación que cursan la formación en modalidad virtual. Estos estudiantes pertenecen a diferentes regiones de Chile, al momento de ser entrevistados rinden el Examen Final de la asignatura Seminario de Grado vía streaming, para finalizar su proceso de estudio. Todos ellos han cursado y aprobado la totalidad de las asignaturas de la formación y con ello han sobrellevado los desafíos que pudieron haber presentado en esta modalidad virtual de educación.

\section{Instrumento de recogida de la información}

Se selecciona la entrevista, ya que corresponde fundamentalmente una conversación en la que y durante la que, se ejercita el arte de formular preguntas y escuchar 
respuestas. En el caso de esta investigación, se considera las respuestas a la entrevista semiestructurada, recogidas para abordar las preguntas en cuestión, se elaboran supuestos descriptivos e interpretativos con el fin de otorgar un sentido a una situación y no de establecer una relación causal lineal en un sentido único. La entrevista semiestructurada se usa cuando el investigador sabe algo acerca del área de interés, por ejemplo, desde la revisión de la literatura, pero no lo suficiente como para responder las preguntas que se ha formulado (Mayan, 2001, p. 16). Este tipo de entrevista puede diseñarse como una entrevista de tópico, es decir, una entrevista que busca indagar sobre determinados evento o tema, en este caso el impacto laboral de la licenciatura en educación a través de la educación virtual.

\section{Resultados}

\section{Sobre la Licenciatura en Educación}

A grandes rasgos, la Licenciatura en Educación corresponde a un período de complementación curricular dirigida a personas que ya se han graduado de otra formación y que pretenden profundizar sus conocimientos teóricos y metodológicos en el campo de la educación, con el fin de ampliar sus conocimientos y competencias para su quehacer educativo.

En este sentido, es necesario comprender el término de Licenciatura en Educación, que según los lineamientos establecidos en el proyecto formativo en la Universidad la República, específicamente en la modalidad virtual, se plantea como objetivo, complementar la formación profesional en el ámbito pedagógico e incorporarse en procesos educativos en los que se reconozcan diversos contextos socio-culturales, desde una perspectiva de inclusión, no discriminación y derechos.

Es posible relacionar esta definición con lo que señalan en su mayoría los estudiantes entrevistados, que entre sus declaraciones, afirman que la Licenciatura en Educación y sus contenidos, fueron complementarios a su formación profesional previa y lo más importante, a las funciones en las que se desempeñan actualmente en los distintos establecimientos educacionales a lo largo del país desde niveles prebásicos hasta en enseñanza media. Sumado a esto, es posible evidenciar a través de algunos relatos que esta formación les ha entregado más herramientas en relación al abordaje de situaciones educativas. A continuación, se presentan algunas opiniones que se relacionan con lo anterior:

"Estudiar licenciatura en educación significó aprendizaje y perfeccionamiento. Todo lo aprendido me ha servido para cambiar ideas erradas y fortalecer mi posición frente a los estudiantes. Aprender técnicas, planificar y muchas otras cosas, ha sido un logro 
personal completo". (Estudiante nro. 18)

"Trabajo en un sector complicado donde es necesario aprender cosas nuevas todo el tiempo para encantar a los estudiantes. Esta licenciatura tiene contenidos precisos y muy completos". (Estudiante nro. 1)

"Todo esto fue necesario para mejorar mi papel como profesora en la sala de clases, era lo que me faltaba, mis actividades eran entretenidas pero les faltaba... ahora son completas". (Estudiante nro. 7)

También es posible evidenciar que los aprendizajes señalados por los estudiantes, les han permitido adquirir conocimientos nuevos y que ellos destacan pues han sido significativos y necesarios. Esto se evidencia a través de las siguientes afirmaciones:

"No sabía planificar y aprendí a hora con la licenciatura. Con esto he aprendido a trabajar de mejor manera, en la elaboración de clases, el trato con mis alumnos, aprender a investigar y nuevas estrategias". (Estudiante nro.10)

"Me permitió comprender el porqué de las prácticas docentes, por ejemplo la necesidad de abordar los conocimientos previos, y otros temas que antes no entendía por qué debía hacerlos". (Estudiante nro. 11)

"Complementa mi aprendizaje con lo que yo tenía, si bien tenía la práctica con niños, esto me ayudó en la organización y análisis de los resultados de las actividades, dentro y fuera de la sala". (Estudiante nro. 14)

Por otra parte, se destacan los procesos en los que debido a esta formación y sus contenidos, la mayoría de los egresados se han integrado con éxito, participando en consejos de profesores e incluso en proyectos y propuestas pedagógicas.

"Estudiar licenciatura en educación me permite hablar más en consejos de profesores por ejemplo, y ahora entiendo más lo que hablan los demás también”. (Estudiante nro. 29)

"Estudié la licenciatura para entregar más herramientas a mis estudiantes, ha resultado mejor de lo que esperaba, me ha permitido posicionarme de mejor manera incluso en consejos de profesores". (Estudiante nro. 49)

Por último, se destacan algunas opiniones que comparan esta licenciatura con otras que corresponden a otras casas de estudio, donde sus contenidos fueron elogiados:

"Estudié la licenciatura para complementar y actualizar conocimientos, me ha gustado mucho. Antes estudié Licenciatura en Educación presencial en la Universidad (...) pero los contenidos eran muy generales, y menos dirigidos a lo que necesitaba concretamente en mi trabajo. Hoy al estudiar esta formación termino contenta porque 
todo me ha servido para mi labor con los niños". (Estudiante nro. 50)

\section{La Educación Virtual}

Según Rama (2019), la virtualización de la educación constituye la mayor innovación actual de los procesos educativos y una de las tendencias más fuertes a escala global (p.45). Es así como la tecnología se ha transformado en un componente dinámico de cambio en la educación a distancia, lo que ha llevado que sistemas universitarios sufran modificaciones con nuevos paradigmas educativos, nuevos actores, otras formas de gestión y también lógicas económicas. En los últimos años, este rápido avance de las Tecnologías de la Información y la Comunicación han permeado los múltiples escenarios y formas de abordar los procesos de enseñanza-aprendizaje en educación superior (Serrano \& Narváez, 2010, p.42)

Actualmente, desde cualquier lugar es posible acceder a estudiar alguna formación académica a través de la educación virtual. Por ende, esta opción de enseñanza se presenta como una oportunidad de realizar plenamente el derecho a la educación, "el acceso a la educación virtual es realmente un bien público ya que no es excluyente: el consumo por parte de una persona no excluye a otras" (Rama, 2019, p. 98). Este derecho implica acceder a las obras intelectuales en la web, la posibilidad de estudiar en cualquier lugar a través de internet, ingresar a bibliotecas digitales, entre otras.

En nuestro país, a partir de la pandemia, y el cambio de modalidad en educación, se han evidenciado dificultades al respecto. Juan Silva (2020), académico de la Universidad de Santiago, señala en una entrevista al periódico virtual chileno El Mostrador, que "en este contexto, los sistemas educativos en todos sus niveles no están preparados para la educación a distancia. Los docentes se formaron para dar clases en forma presencial, no online, además no se capacitaron". Considerando esta afirmación, ¿cómo es posible brindar una educación en modalidad virtual que responda a lo significativo de los aprendizajes esenciales de la Licenciatura en Educación?

\section{Acerca de los facilitadores relacionados con la metodología de educación virtual}

Si bien la educación virtual está presente desde hace muchos años en Chile, el desarrollo de grandes redes digitales ha permitido extenderlo hasta muchos lugares antes inaccesibles, lo que ha permitido que aquellas personas que no tenían acceso a la educación hoy sí la tengan. En este sentido, la educación presencial tiene limitaciones pues no siempre corresponde a un derecho al que todos pueden 
acceder, considerando las zonas más rurales e inhóspitas de nuestro país, incluso en las limitaciones de libertad, o de movilidad constituye limitaciones para el derecho a la educación presencial. La educación virtual, en tanto llega a los hogares, en los momentos en que las personas pueden, o en los lugares donde tienen la posibilidad, convirtiéndola en una opción más amplia y democrática ya que se ajusta a las particularidades de las personas (Rama, 2019, p. 97). Es posible evidenciar lo anterior en algunos extractos de las respuestas de los estudiantes:

"Me acomodó bastante, sobretodo porque vivo en un pueblo pequeño en la sexta región, donde es difícil moverse para estudiar y también a veces se complica la señal" (Estudiante nro. 47)

"Que sea online me facilitó mucho. Por los horarios de mi trabajo no alcanzaba a llegar a clases presenciales, y me acomodó mucho también por mi familia".

(Estudiante nro. 39)

"Puedo controlar y aprovechar mis tiempos, alcanzo a hacer todo en el día, es menos estresante que ir a clases presenciales". (Estudiante nro. 32)

"No salir de mi casa me permitió estar con mis hijos en situaciones complejas. Ser responsable de mi aprendizaje me ayudó a regular mis tiempos." (Estudiante nro. 26) "Fue lo mejor, muy cómodo y completo. Podía responder a lo que me pedían desde cualquier parte con mi notebook". (Estudiante nro.20)

Otro aspecto positivo evidenciado en las respuestas de los estudiantes consultados es en relación a los materiales utilizados en las asignaturas, lo que es posible observar en los siguientes extractos:

"Lo bueno es que todos los textos estaban en internet, no tenía que buscar libros ni sacar fotocopias" (Estudiante nro. 29)

"Consulté muchas veces la bibliografía y habían cosas bien interesantes que motivaban a seguir investigando." (Estudiante nro.44)

"Compartí con mis compañeros y hasta hicimos trabajos juntos." (Estudiante nro.34)

"..., los contenidos, las evaluaciones permitían trabajar sola o en grupo, lo que era bueno para conocer a los compañeros" (Estudiante nro. 21)

Considerando el punto anterior, fue posible detectar algunas experiencias de estudiantes que manifestaron su parecer en relación a la pertinencia de las respuestas de los docentes. He aquí algunos extractos de lo señalados por los estudiantes consultados:

"Todos los profesores manejaban muy bien los temas y respondían las dudas en forma clara" (Estudiante nro. 19) 
"Gracias a los profesores que siempre estuvieron pendientes y respondiendo los mensajes..." (Estudiante nro. 22)

"Hay profesores que estuvieron pendientes en todo momento." (Estudiante nro. 33)

"Buena comunicación entre estudiantes y profesores." (Estudiante nro. 37)

"Me acomodó bastante esta modalidad, hay mucha fluidez en la comunicación, claridad en los videos y prontitud en las respuestas de los docentes y la coordinación." (Estudiante nro. 40)

"Los profesores fueron conscientes ante dificultades y muy rápidos en sus respuestas." (Estudiante nro. 43)

\section{Las barreras que experimentaron los estudiantes en la modalidad virtual}

Una de las principales barreras evidenciadas corresponde a algunas situaciones en que los docentes han respondido con tardanza a las preguntas de los estudiantes enviadas por mensajería en el Aula Virtual, lo que a veces ocurre luego de finalizado el plazo de entrega de evaluación correspondientes a un módulo o clase trabajada. Esta situación muchas veces llega a manos de la coordinación, donde es posible plantear otras medidas para dar oportunidades de respuesta a los estudiantes, pues en estricto rigor, al no tener respuesta del profesor, tienen dos alternativas: enviar el trabajo a partir de su propia respuesta a la inquietud; o bien, no enviarlo, pues no queda claro qué es lo que deben hacer. Pese que se entregan oportunidades posteriores, esto podría traducirse como un aprendizaje incompleto, e incluso en una posterior falta de motivación para el estudiante.

En este aspecto, el trabajo de los coordinadores académicos de las formaciones es importante pues permite dejar en evidencia si efectivamente se está llevando a cabo de forma adecuada este proceder. Algunos extractos de las respuestas de estudiantes que evidencian el punto anterior son las siguientes:

"A veces algunos profesores se demoraban en responder dudas, otras veces yo me demoraba en hacerlas." (Estudiante nro. 14)

"En ocasiones los docentes no respondían a tiempo, no cumpliendo los plazos, lo que mejoró después." (Estudiante nro. 15)

"La respuesta a las preguntas es más lenta que en presencial, pese a eso siempre hubo una buena orientación de los profesores." (Estudiante nro. 30)

"Algunos profesores responden a las dudas tarde." (Estudiante nro. 34)

"A veces había profesores que se demoraban en la entrega de notas, pero uno avisaba y se apuraban." (Estudiante nro. 10)

"A veces había preguntas a profesores con respuestas más lentas..." (Estudiante nro. 46) 
Junto a esta situación es necesario tomar en cuenta otros factores que pueden ser considerados como barreras inicialmente para los estudiantes y su proceso de aprendizaje.

"Esta modalidad me pone más nerviosa que la presencial, porque no veo a las personas..." (Estudiante nro. 35)

"Al principio me asusté por mi edad..." (Estudiante nro. 41)

"Me obligó a ser más disciplinada y a perder el temor de hablar en los videos..." (Estudiante nro. 42)

"Estudiar por computador significó otras obligaciones, otras responsabilidades... De principio había miedos..." (Estudiante nro. 2)

Es necesario tomar en cuenta el carácter ambivalente de la tecnología, es imposible no considerar los aspectos negativos que conlleva, por ejemplo, las condiciones materiales para desarrollar una actividad virtual, donde es necesario disponer en los hogares de ciertos recursos como equipos adecuados, conexión estable, conocimientos relacionados con el uso del equipo y software, aplicaciones descargadas, entre otros aspectos. Este tipo de barreras son definidas como situacionales, y son producto de la situación general del individuo y su contexto. Incluye entre otros elementos la edad, las restricciones de tiempo, las responsabilidades familiares, etc. También se consideran barreras disposicionales, considerando aquellas resultantes de la experiencia individual, incluyendo actitud, motivación, estilos de aprendizaje y autoconfianza. (Feierherd \& varios, 2003, p. 1276)

\section{Discusión}

Los resultados generados a partir de la entrevista, logran identificar el impacto de la formación en Licenciatura en Educación, como complemento curricular a personas que pretenden profundizar sus conocimientos teóricos y metodológicos en el campo de la educación, con el fin de ampliar sus conocimientos y competencias para su quehacer educativo, superando incluso las expectativas de algunos.

Dentro de las respuestas de los estudiantes, se destaca no solamente el significado de estos nuevos conocimientos en el trabajo o en la organización con sus alumnos en la planificación, implementación y evaluación de actividades, sino también el empoderamiento que se evidencia en la experiencia de algunos de los profesionales que son parte de una comunidad educativa. Por otra parte, es importante destacar la invitación a reflexionar y argumentar que les proporciona la Licenciatura en Educación, donde los estudiantes señalan que les ha permitido identificar y analizar con argumentos teóricos aspectos deficientes del sistema educativo, a través del 
conocimiento de leyes, y de la importancia de la inclusión en diversos contextos escolares, por medio de contenidos relacionados con la historia de la educación, psicología, ética profesional, entre otros.

A partir de lo anteriormente expuesto, es posible afirmar que se cumple el primer supuesto, es decir, la Licenciatura en Educación contribuye de forma positiva al desarrollo integral de los estudiantes que la cursan, ya que según se evidencia en sus respuestas, éstos reciben herramientas adecuadas que les permiten desenvolverse en su labor en establecimientos educacionales, potenciando el desarrollo de las habilidades en relación a los estudiantes con quienes trabajan y con otros docentes, respecto a conocimientos del quehacer pedagógico.

Hodges y colaboradores (2020) respecto a la educación e-learning, señalan que si bien la educación a distancia en general carga con un estigma de menor calidad, esta se desarrolla en universidades prestigiosas y ha demostrado importantes avances en la última década. La educación a distancia descansa en un diseño y planificación cuidadosos con vasta evidencia y se rige por indicaciones instruccionales definidas.

Considerando lo anterior, Rama (2019, p. 72), plantea una propuesta de modelo educativo desarrollada en Argentina, que se ha conformado en torno a la educación virtual, y está relacionada con diversos ejes de regulación como son: 1) Una efectiva dinámica de interacción docente-estudiante y estudiantes entre ellos; 2) Materiales especiales para la enseñanza; 3) Requisitos mínimos de niveles de formación docente; 4) Tecnologías digitales de información y comunicación; 5) Un formato de evaluación; y 6) El funcionamiento de sedes de apoyo.

Al analizar cada uno de los ejes planteados por el autor y compararlos con los resultados de esta investigación obtenidos, es posible observar lo siguiente:

Respecto a la consideración de una efectiva dinámica de interacción docenteestudiante y estudiantes entre ellos, los lineamientos de la Universidad La República, proponen que el docente ejercerá su quehacer a través del diálogo pedagógico con sus estudiantes, favoreciendo el pensamiento crítico tanto del contenido de la disciplina como de las propias prácticas y/o didácticas. Es un guía activo, facilitador y transformador, comprometido con una sociedad más laica, tolerante, humanista y republicana.

Es posible señalar que en la modalidad virtual existe el diálogo que se menciona en el párrafo anterior, y éste logra favorecer el pensamiento crítico a través de las diversas actividades que posee cada módulo de una asignatura, donde se promueve la opinión de los estudiantes a través de foros de discusión y evaluaciones. A esto se suma también la comunicación que existe entre profesores y estudiantes, a través de la 
mensajería por medio de la plataforma. Una de las barreras que se alertan es la tardanza de las respuestas de algunos profesores a dudas y calificaciones, situación que es posible observar a tiempo si se realiza una revisión de los accesos de los docentes al Aula Virtual, este trabajo que se da en conjunto de coordinación académica y la encargada de la plataforma. Es necesario informar de inmediato al docente. Pero, ¿por qué los profesores tardan en responder a los estudiantes? Al consultar a los docentes por esta situación que no ocurre con regularidad en las asignaturas, los profesores señalan que no alertaron la situación a tiempo porque tienen otros trabajos, o bien que no están acostumbrados aún a esta modalidad no presencial. Por esta razón, se hace imprescindible indicar inicialmente y reiterar en el proceso a los docentes, que deben revisar la plataforma y responder a las inquietudes en un máximo de 24 horas, luego de formulada la pregunta.

En relación con elaboración de materiales especiales para la enseñanza virtual, cada módulo de una asignatura posee recursos entregados por los docentes como la presentación PowerPoint, la pregunta de foro de discusión, la guía del estudiante, la evaluación del módulo, la bibliografía obligatoria y complementaria, y la ruta del módulo. Cada uno de estos recursos es revisado cuidadosamente por coordinación académica y luego validado por la diseñadora instruccional de la formación. Todos poseen criterios para su elaboración y deben siempre abordar los contenidos dispuestos en el programa de la asignatura. Cabe destacar que la bibliografía cuenta con links de acceso, de esta forma, los estudiantes no deben acudir obligatoriamente a bibliotecas u otros lugares para acceder a la bibliografía, sólo deben contar con la conexión a internet y así no interrumpir sus estudios, lo que ha sido valorado por los estudiantes que cursan esta formación.

Por otra parte, respondiendo a los requisitos mínimos de niveles de formación docente, cada programa entrega los requisitos de los docentes que estarán encargados de las asignaturas, una vez que se identifica el perfil se busca coincidencia con el postulante a través de la revisión de su currículum y una entrevista, dejando claridad desde un primer momento de las diferentes exigencias que tiene esta modalidad y el rol que deben cumplir como docentes de esta formación. Sin embargo, según el documento de la Comisión Nacional de Acreditación denominado Orientaciones para la acreditación de instituciones que imparten programas en modalidad virtual y combinada (2017), deberían además existir programas de capacitación que permitan al personal directivo, académico y administrativo atender los requerimientos metodológicos y tecnológicos necesarios para la implementación de la educación virtual. Situación que se encuentra aún en proceso de ser desarrollada en la Universidad La República. 
Al hablar de las tecnologías digitales de información y comunicación, cabe señalar que posible contar con infraestructura tecnológica adecuada, donde se dispone de servicios informáticos y conectividad, que dan garantía a la calidad y continuidad de la implementación del programa utilizado en la educación virtual.

Respecto al formato de evaluación, es posible señalar que según los lineamientos de la Universidad La República, los sistemas de evaluación utilizados permiten al docente acompañar al estudiante en la construcción de su conocimiento, monitorear el nivel de logro de los aprendizajes y verificar la calidad de los desempeños que contribuyen a la utilización de métodos de evaluación de los conocimientos y de las capacidades adquiridas por los estudiantes. En este aspecto, es posible destacar la rigurosidad que existe en cada asignatura en relación a la creación o las posibles modificaciones que se pretendan realizar a los recursos correspondientes. En estricto rigor, cada asignatura está compuesta por tres módulos (excepto Metodología de la Investigación que tiene cuatro, y Seminario de Grado que posee cinco), en ellos existen tres tipos de evaluación diferentes: la primera, un control de contenidos con preguntas de selección múltiple y con verdadero y falso, la segunda corresponde a un trabajo individual y la tercera evaluación se refiere a un trabajo grupal. Se define inicialmente de esta manera, con el propósito de entregar más variedad en las formas de evaluación, fomentando la motivación en este proceso y de promover que los estudiantes puedan intercambiar ideas entre sí, y que conozcan a sus compañeros virtualmente, complementando el objetivo del foro de discusión donde podrían realizar esta acción igualmente.

Un punto que se encuentra en desarrollo en esta formación y su modalidad es el funcionamiento de sedes de apoyo. En definitiva, la educación virtual debe contar con estándares de evaluación similares que la educación presencial, ya que de igual manera deben pasar por procesos de evaluación. Según el documento de la Comisión Nacional de Acreditación denominado Orientaciones para la acreditación de instituciones que imparten programas en modalidad virtual y combinada (2017), además de una estructura institucional dedicada a la entrega de apoyo pedagógico, administrativo, de gestión y de soporte técnico, deberá existir una estructura institucional que entrega soporte socio-afectivo a los procesos de enseñanza y aprendizaje desarrollados por medio del entorno virtual de aprendizaje. Este aspecto es el que se ve más descendido en esta modalidad, pues esta casa de estudios cuenta una sede de apoyo, tanto para modalidad virtual como presencial, que se encarga de aspectos como el apoyo psicológico, social, en manejo de stress, dificultades de aprendizaje, entre otros aspectos.

A partir del análisis de los párrafos anteriores, es posible afirmar que se cumple con 
el segundo supuesto, pues la modalidad virtual impartida en esta formación, brinda las oportunidades para un adecuado manejo de los contenidos tratados en cada asignatura y módulo de éstas, otorgándoles herramientas a través de una metodología de estudio que responde a sus intereses y necesidades de manera íntegra. Sin embargo, es posible observar aspectos que aún se encuentran en vías de desarrollo, considerando el abordaje de las particularidades de cada estudiante, aspectos que se han transformado en barreras como son las respuestas tardías a las inquietudes de los estudiantes, lo que ocurre esporádicamente, empero pueden existir consecuencias negativas en el proceso de aprendizaje de dicho estudiante.

A través de los lineamientos de la Universidad La República, es posible observar que se cumplen los supuestos planteados a partir de la formación que se imparte a través de la Licenciatura en Educación, pues en muchas de las respuestas de los egresados se puede evidenciar que adquirieron los conocimientos de su disciplina, a través del desarrollo de competencias por medio de la modalidad virtual.

Recapitulando, es posible identificar como adecuada y favorable la experiencia de este grupo de estudiantes que cursaron Licenciatura en Educación a través de la modalidad virtual, esto en base a sus opiniones y vivencias en relación a la formación y a esta modalidad. Esta experiencia se convierte en un impulso a continuar investigando en el área de educación virtual que, si bien lleva varios años desarrollándose en la sociedad chilena, cada día es posible encontrar más opciones educativas y más personas que la eligen, sobre todo considerando la situación de pandemia actual. Ante esto, debe existir una mirada objetiva que permita revisar y cuestionar la implementación de esta modalidad virtual de manera crítica y constructiva, con el fin de promover que se imparta una educación de calidad, y que ésta cumpla con los objetivos referentes a participación, respeto, valoración de los estudiantes y docentes, además del logro de objetivos de aprendizaje.

\section{Referencias}

Angrosino, M. (2012). Etnografía y observación participante en investigación cualitativa. Ediciones Morata.

Baptista, M., Hernández, R. y Baptista, M. (2014). Metodología de la investigación (6a. ed.). McGraw-Hill Interamericana.

Comisión Nacional de Acreditación (2017). Orientaciones para la acreditación de instituciones que imparten programas en modalidad virtual y combinada.

Feierherd, G. \& varios (2003) Las barreras en la educación superior no presencial. Grupo de Investigación en Tecnologías Informáticas Aplicadas (GITIA). Facultad de 
Ingeniería - Sede Ushuaia. CORE. https://core.ac.uk/download/pdf/15779663.pdf

Fundación Universitaria Católica del Norte (2005) EDUCACIÓN VIRTUAL. Reflexiones y Experiencias. UCN.

https://www.ucn.edu.co/institucion/sala-prensa/Documents/educacion-virtualreflexiones-experiencias.pdf

Mayan, M. (2001) Una introducción a los métodos cualitativos. Qual Institute Press International Institute for Qualitative Methodology. Ualberta. https://sites.ualberta.ca/ iiqm/pdfs/introduccion.pdf

Nieto, R. (2012) Educación virtual o virtualidad de la Educación. Revista Historia de la Educación Latinoamericana, 14,19. Universidad Pedagógica y Tecnológica de Colombia. pp. 137-150.

Rama, C. (2019) Políticas, tensiones y tendencias de la EDUCACIÓN A DISTANCIA Y VIRTUAL en América Latina. Ediciones Universidad Católica de Salta.

Rodríguez, J. (2004) El aprendizaje virtual. Homosapiens Ediciones.

Serrano J. \& Narváez P. (2009) Uso de software libres para el desarrollo de contenidos educativos.

Researchgate. https://www.researchgate.net/publication/262544570_Uso_de_Software_Libre_par a_el_Desarrollo_de_Contenidos_Educativos

Sierra, C. (2011) La educación virtual como favorecedora del aprendizaje autónomo. Revista Panorama N 9. Institución Universitaria Politécnico Grancolombiano. Pp. 75 87. Dialnet. https://dialnet.unirioja.es/descarga/articulo/4780035.pdf

\footnotetext{
María Angélica Sanhueza Álvarez

Profesora de Educación Diferencial, mención en Problemas de Aprendizaje. Universidad Metropolitana de Ciencias de la Educación. Magíster en Educación, mención Gestión Educacional. Universidad Metropolitana de Ciencias de la Educación Docente Universidad Central, Modalidad Presencial. Coordinadora Académica Licenciatura en Educación, Modalidad Virtual. Directora Magíster en Gestión y Liderazgo Educacional, Modalidad Virtual. Universidad La República.
} 